\title{
A Novel Protocol for Network-Controlled Metasurfaces
}

\author{
Angeliki Tsioliaridou \\ FORTH, Greece \\ atsiolia@ics. forth.gr \\ Christos Liaskos \\ FORTH, Greece \\ cliaskos@ics.forth.gr \\ Andreas Pitsillides \\ University of Cyprus, Cyprus \\ Andreas.Pitsillides@ucy.ac.cy \\ Sotiris loannidis \\ FORTH, Greece \\ sotiris@ics.forth.gr
}

\begin{abstract}
A recently proposed class of materials, called software-defined metamaterials, can change their electromagnetic behavior on demand, utilizing a nanonetwork embedded in their structure. The present work focuses on 2D metamaterials, known as metasurfaces, and their electromagnetically programmable counterparts, the HyperSurfaces. The particular focus of the study is to propose a nanonetworking protocol that can support the intended macroscopic functionality of a HyperSurface, such as sensing and reacting to impinging waves in a customizable manner. The novel protocol is derived analytically, using the Lyapunov drift minimization approach, taking into account nano-node energy, communication latency and complexity concerns. The proposed scheme is evaluated via simulations, covering both the macroscopic HyperSurface functionality and the microscopic, nanonetwork behavior.
\end{abstract}

Keywords: Electromagnetic nano-networking, metasurfaces, protocol.

${ }^{*}$ This work was funded by the European Union via the Horizon 2020: Future Emerging Topics call (FETOPEN), grant EU736876, project VISORSURF (http://www.visorsurf.eu). 


\section{Introduction}

Extending the reach of communications, control and networking to the nano-meter level offers unprecedented applications in sectors such as medicine and material manufacturing [13, 1]. Targeted drug delivery at cellular level and sensing for material manufacturing flaws at nano-scale are among the envisioned tasks. Recently, another application of nanonetworks has been proposed, in the form of objects with programmable electromagnetic behavior [10]. These objects emerge as the combination of metasurfaces-a discipline of Physics-and nanonetworks, offering novel potential in the interaction with electromagnetic waves.

Metasurfaces are planar, artificially structured objects that can interact with impinging electromagnetic waves even in ways not found in nature [4. They commonly comprise a two-dimensional pattern of a conductive material, the meta-atom, repeated periodically over a dielectric substrate. The form and dimensions of the meta-atom exemplary define the reflection angle-even at negative values-and absorption degree of impinging waves. The HyperSurface concept takes the metasurface concept one step further: it is a hardware platform that allows for forming custom meta-atoms over it, with local or global periodicity [15]. A nanonetwork embedded within the HyperSurface acts as the meta-atom control factor. It receives external commands and "draws" meta-atoms by altering the local conductivity of the HyperSurface accordingly.

The present paper treats the HyperSurface as a network-controlled system, and proposes a novel protocol (Hyper-CP) for its internal operation. The protocol can carry sensed information to the external world for processing, as well as HyperSurface configuration commands from the external world to the HyperSurface. Hyper-CP relies on Lyapunov drift analysis to provide natural path-diversity to avoid congested or out-of-power nanonetwork areas [3]. Simulations are employed to evaluate Hyper-CP, which consider both the microscopic (nanonetwork operation) and the macroscopic (detecting impinging waves) aspects of the HyperSurface. Thus, adapting the HyperSurface electromagnetic configuration is also enabled.

The remainder of this paper is organized as follows. Section 2 provides the necessary background knowledge and the system model. Section 3 details the proposed, HyperSurface-compatible networking scheme. Evaluation via simulations takes place in Section 4. Related studies are given in Section 5 and the conclusion is given in Section 6

\section{Background and System Model}

Background. Metasurfaces are the outcome of a research direction in Physics that seeks to construct objects with engineered electromagnetic behavior. In their most classic form, metasurfaces are composed of a building block (meta-atom) that is periodically repeated over a given area. When this periodic structure is treated at a macroscopic level, the metasurface as a whole can exhibit custom and even exotic permeability and permittivity values. This in turn translates to custom-and even unusual-interaction between the metasurface and impinging electromagnetic waves. For instance, a metasurface can exhibit an unnatural, negative refractive index, causing light to impinging waves to bend backwards, at a negative angle of refraction. To understand the impact of this potential, we mention that a carefully designed stack of metasurfaces, each with its own refraction angle, was able to render an object invisible to EM waves (cloaking) by gradually bending them around it 5 . 

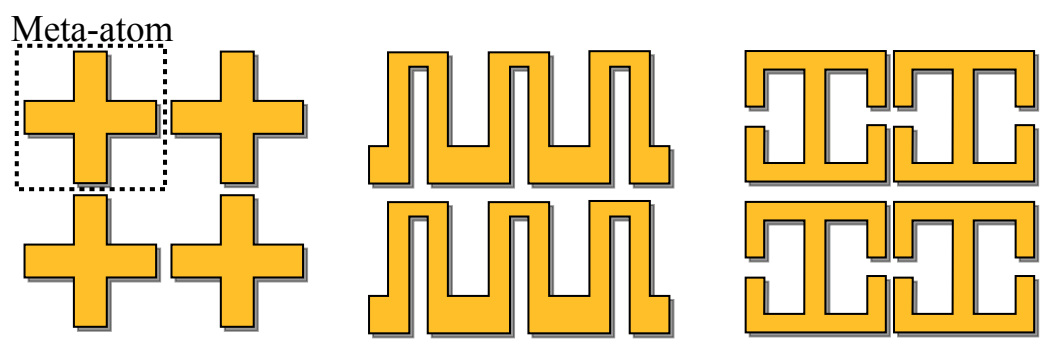

Fig. 1: Indicative meta-atom patterns that have been employed in metasurface studies.

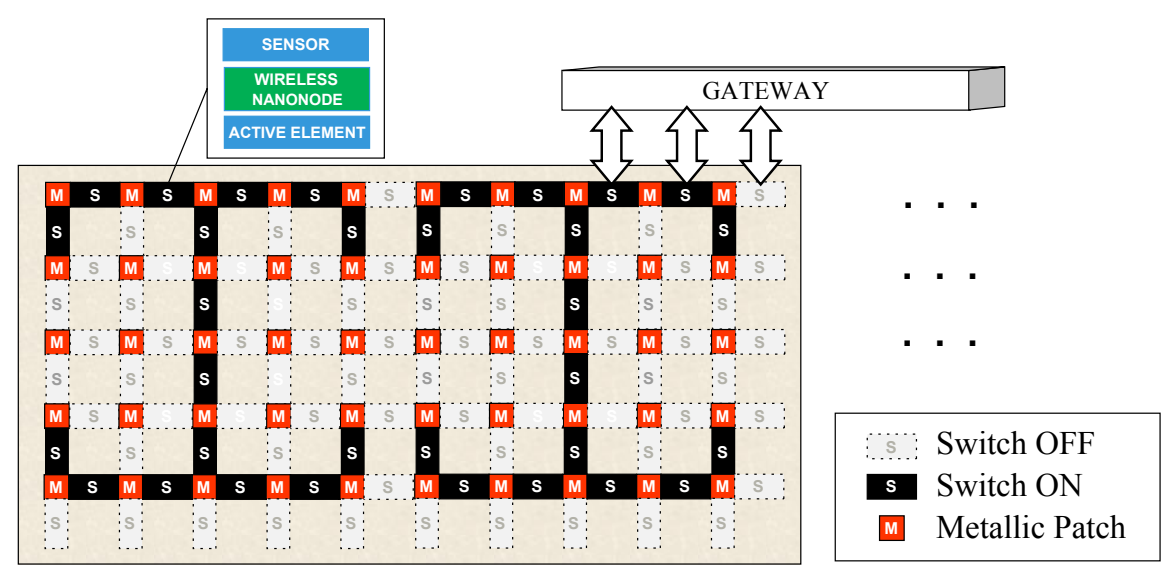

Fig. 2: The HyperSurface structure, comprising passive elements (patches), active elements (binary switches) and networked nano-controllers.

Some common meta-atom patterns are shown in Fig. 1. The meta-atom patterns are usually composed of a conductive material and are placed over a dielectric substrate. Copper and Silicon constituted very early material choices, while many more options, such as Graphene, have been studied since [23]. One of the most commonly studied, macroscopic interactions between a metasurface and impinging electromagnetic waves, is wave steering [11. It expresses the reflection of an impinging wave from a given incident angle to a direction dependent on the meta-atom configuration.

A HyperSurface is a network-controlled metasurface. Its core is a hardware layer that can change its internal structure to best fit an intended meta-atom deployment. In general, this layer comprises passive and active elements. The formation of programmatic meta-atom arrangements is achieved by tuning the state of the active elements accordingly. A simple example is shown in Fig. 2, comprising metallic patches and CMOS switches. Custom meta-atoms are stitched together by setting the switches appropriately to ON (conductive) or OFF (insulating) states.

The system model. We assume that the state of each single active element is managed by a nano-controller (node). Each controller is placed under a metallic 


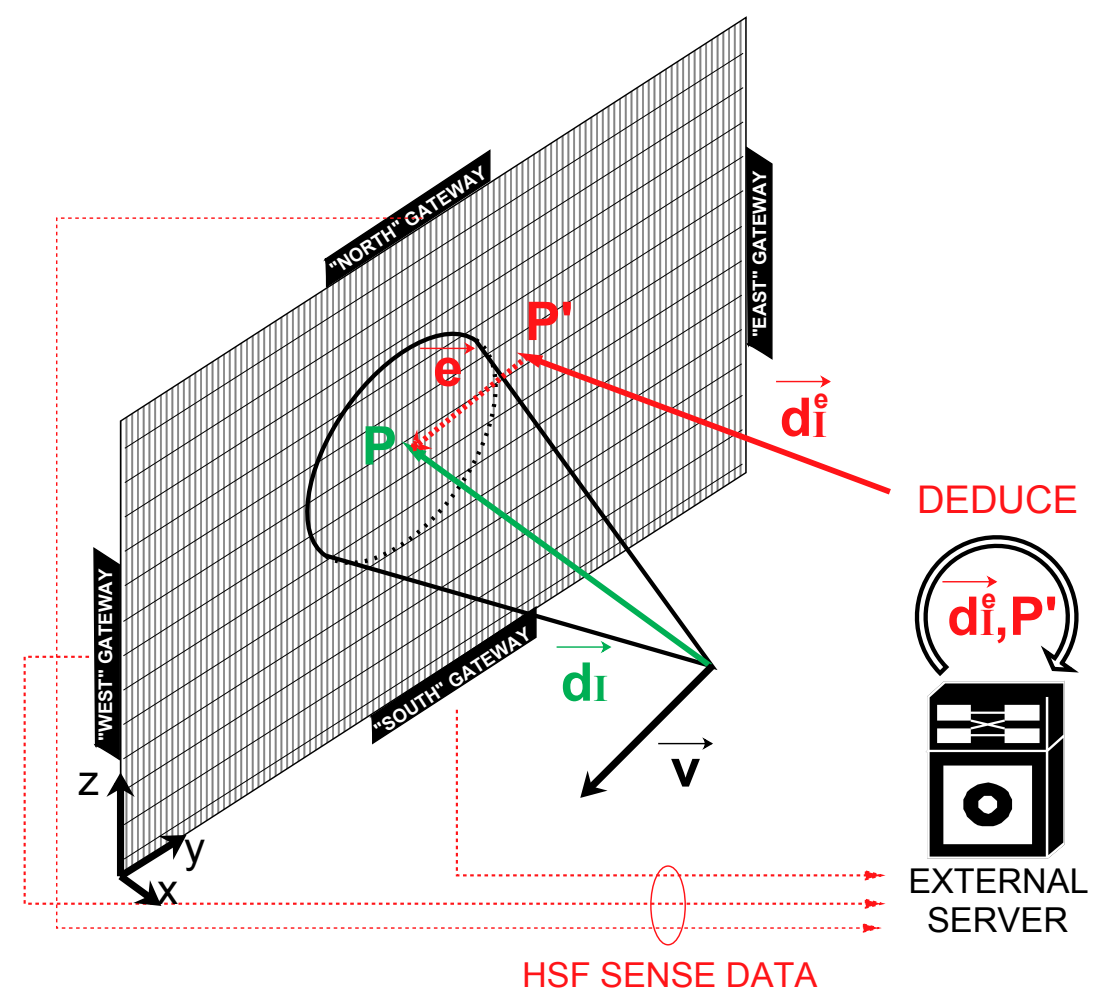

Fig. 3: HyperSurface end-functionality and interconnectivity with external networked entities.

patch, to shield it from impinging eaves. Additionally, it has wired connectivity to its immediate neighbors, thus forming a rectangular grid network topology overall. Each node has a hard-coded, known position over the HyperSurface in the form of $i, j$ coordinates, which also act as the node's unique address.

The node power supply relies on energy-harvesting, charging a battery of limited capacity. Each node has the ability to sense the power level of impinging waves on the HyperSurface at their location. A sensor measurement is captured in the form of a data packet, and is stored on a small buffer, awaiting forwarding to the HyperSurfaceexternal world, via multi-hop transmission towards one or more gateways. The gateway configuration is considered to be known by design. All nodes are identical, and a packet is assumed to be delivered once it has reached on of the nodes adjacent to a gateway.

To facilitate the routing process, any packet about to be transmitted (original or retransmission) carries statistics regarding the status of the present node. Some specific statistics are discussed in Section 3 An immediate neighbor reads this information and utilizes it to optimize routing decisions, e.g., by extrapolating the statistics to obtain the current neighborhood status, without exchanging specific control packets for this task. 
Figure 3 illustrates a complete HyperSurface loop comprising a mobile wave source, and an external server that receives the sensed data to yield the estimated, time-variant incident direction $\overrightarrow{d_{I}^{e}}$ and impact point $P^{\prime}$. Network latency introduces a direction error, expressed by the formation of an angle, $\Theta^{e}$, between the real direction, $\overrightarrow{d_{I}}$, and its estimation, $\overrightarrow{d_{I}^{e}}$, as well as an offset error, $\vec{e}$, defined by the real impact point, $P$, and the estimated, $P^{\prime}$. Our goal is to study the effects of a nanonetworking data routing protocol on the direction and offset errors.

\section{The Hyper-CP Protocol}

The proposed HyperSurface Control Protocol (Hyper-CP) seeks to route a continuous stream of sensed data to the external world, taking into account energy and latency considerations. Ideally, sensed data should be routed towards the gateways over the shortest routes. However, as congestion increases and node batteries get depleted, alternative paths should be naturally followed. To accomplish these objectives, Hyper-CP relies on the concept of Lyapunov drift minimization, which is well-known to yield network throughput-optimality (i.e., natural path diversity when needed), and extremely simple routing criteria [3]. We complement this process by assuming outdated node status info, and battery/latency considerations.

The Lyapunov drift of a network is a time variant quantity defined as [3]:

$$
L(t)=\sum_{n} \sum_{w} U_{(n, w)}^{2}(t)
$$

where $n, w \neq n$ are network nodes, and $U_{(n, w)}(t)$ is the current data buffered at node $n$ and destined towards node $w$. We shall define an upper bound for $L(t)$ in the HyperSurface case, and then proceed to minimize it.

We begin by expressing the estimated queue size of a single node at time $t$ as:

$$
\underset{(n, w)}{U^{e}(t)=\max \left\{0, \underset{(n, w)}{U}\left(t-\tau_{n}\right)-O_{(n, w)}^{t-\tau_{n} \rightarrow t}\right\}+} \begin{aligned}
& I_{(n, w)}^{t-\tau_{n} \rightarrow t}+G_{(n, w)}^{t-\tau_{n} \rightarrow t}
\end{aligned}
$$

where $w$ denotes a HyperSurface gateway, $\tau_{n}$ is the point in the past when the real status of node $n$ was reported, $O_{(n, w)}^{t-\tau_{n} \rightarrow t}, I_{(n, w)}^{t-\tau_{n} \rightarrow t}$ and $G_{(n, w)}^{t-\tau_{n} \rightarrow t}$ denote estimated outgoing, incoming and locally generated data (via sensing).

Both sides of equation (2) are squared, and the following identity is applied [3, p. $53]$,

$$
V \leq \max \{0, U-\mu\}+A \Rightarrow V^{2} \leq U^{2}+\mu^{2}+A^{2}-2 U \cdot(\mu-A)
$$

leading to:

$$
\begin{aligned}
\left(\underset{(n, w)}{\left.U^{e}(t)\right)^{2} \leq}{ }_{(n, w)}^{U^{2}}\left(t-\tau_{n}\right)+\right. & \\
& {\left[O_{(n, w)}^{t-\tau_{n} \rightarrow t}\right]^{2}+\left[I_{(n, w)}^{t-\tau_{n} \rightarrow t}+G_{(n, w)}^{t-\tau_{n} \rightarrow t}\right]^{2}-} \\
2 & \cdot \underset{(n, w)}{U}\left(t-\tau_{n}\right) \cdot\left[O_{(n, w)}^{t-\tau_{n} \rightarrow t}-I_{(n, w)}^{t-\tau_{n} \rightarrow t}-G_{(n, w)}^{t-\tau_{n} \rightarrow t}\right]
\end{aligned}
$$

We proceed to replace the right part of relation (2) with an upper bound. Let $\mathcal{R}_{l}$ denote the nominal data rate of a link $l$. Moreover, let $\mathcal{P}_{(n \rightarrow *)}$ and $\mathcal{P}_{(* \rightarrow n)}$ denote 
the set of directed links originating from and ending at node $n$ respectively, while also being compliant to custom selection criteria, denoted as policy $\mathcal{P}$. Then, we write:

$$
I_{(n, w)}^{t-\tau_{n} \rightarrow t} \leq \tau_{n} \cdot \sum_{l \in \mathcal{P}_{(* \rightarrow n)}} \mathcal{R}_{l}, O_{(n, w)}^{t-\tau \rightarrow t} \leq \tau_{n} \cdot \sum_{l \in \mathcal{P}_{(n \rightarrow *)}} \mathcal{R}_{l}
$$

Using the bounds of 5 and setting $\Delta U_{(n, w)}^{2}(t)=\left(\underset{(n, w)}{U}{ }^{e}(t)\right)^{2}-\underset{(n, w)}{U}{ }^{2}\left(t-\tau_{n}\right)$, we obtain:

$$
\begin{aligned}
\Delta U_{(n, w)}^{2}(t) \leq \tau_{n}^{2} & \cdot\left(\sum_{l \in \mathcal{P}_{(n \rightarrow *)}} \mathcal{R}_{l}\right)^{2}+\left[\tau_{n} \cdot \sum_{l \in \mathcal{P}_{(* \rightarrow n)} \mathcal{R}_{l}}+G_{(n, w)}^{t-\tau_{n} \rightarrow t}\right]^{2} \\
& -2 \cdot \underset{(n, w)}{U}\left(t-\tau_{n}\right) \cdot\left[\tau_{n} \cdot \sum_{l \in \mathcal{P}_{(n \rightarrow *)}} \mathcal{R}_{l}-\tau_{n} \cdot \sum_{l \in \mathcal{P}_{(* \rightarrow n)}} \mathcal{R}_{l}-G_{(n, w)}^{t-\tau_{n} \rightarrow t}\right]
\end{aligned}
$$

Inequality (6) can be rewritten as follows:

$$
\begin{aligned}
\Delta U_{(n, w)}^{2}(t) \leq\left[\tau_{n} \cdot \sum_{l \in \mathcal{P}_{(* \rightarrow n)}} \mathcal{R}_{l}+\underset{(n, w)}{U}\left(t-\tau_{n}\right)+G_{(n, w)}^{t-\tau_{n} \rightarrow t}\right]^{2} & \\
+ & {\left[\tau_{n} \cdot \sum_{l \in \mathcal{P}_{(n \rightarrow *)}} \mathcal{R}_{l}-\underset{(n, w)}{U}\left(t-\tau_{n}\right)\right]^{2}-2 \cdot \underset{(n, w)}{U^{2}\left(t-\tau_{n}\right)} }
\end{aligned}
$$

We sum both sides $\forall n, w$, to obtain the Lyapunov drift at the left part:

$$
\begin{aligned}
& \Delta L(t) \leq \sum_{\forall n} \sum_{\forall w}\left[\tau_{n} \cdot \sum_{l \in \mathcal{P}_{(* \rightarrow n)} \mathcal{R}_{l}}+\underset{(n, w)}{U}\left(t-\tau_{n}\right)+G_{(n, w)}^{t-\tau_{n} \rightarrow t}\right]^{2} \\
& +\sum_{\forall n} \sum_{\forall w}\left[\tau_{n} \cdot \underset{l \in \mathcal{P}_{(n \rightarrow *)} \mathcal{R}_{l}}{-\underset{(n, w)}{U}\left(t-\tau_{n}\right)}\right]^{2}-2 \cdot \sum_{\forall n} \sum_{\forall w} \underset{(n, w)}{U^{2}}\left(t-\tau_{n}\right)
\end{aligned}
$$

The terms $\sum \mathcal{R}_{l}$ in the right part can be viewed as the data routing decisions to be taken at time $t$. The following Theorem provides the optimal decisions that minimize the Lyapunov drift bound, i.e., the right part of relation 8.

Theorem. The Lyapunov bound-optimizing routing decision at node $n$, regarding traffic destined to gateway $w$, is to forward the traffic to neighboring node $m^{*}$ :

$$
m^{*}=\operatorname{argmax}_{m \in \mathcal{M}}\left\{\underset{(n, w)}{U}(t)-\left(\underset{(m, w)}{U^{e}}(t)+G_{(m, w)}^{t-\tau_{m} \rightarrow t}\right)\right\}
$$

where $\mathcal{M}$ is the set containing the neighbors of $n$ that comply with policy $\mathcal{P}$.

The proof is omitted.

Theorem 1 dictates a very simple data forwarding loop for the nano-nodes, formalized as Algorithm 1] which is repeated as long as a node is powered and has pending packets in its queue. A node $n$ needs just an estimation of its neighbors $\underset{(m, w)}{U}{ }^{e}(t)$ and $G_{(m, w)}^{t-\tau_{m} \rightarrow t}$ quantities. The latter is the packet generation rate at a node, 


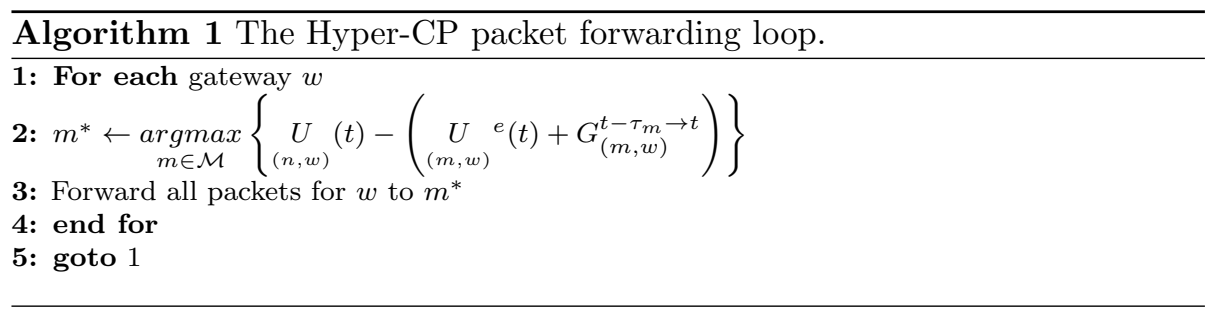

due to its sensing duties. Assuming a global, hard-wired sense rate of $\bar{g}$ (packets per time unit), a node $n$ can estimate $G_{(m, w)}^{t-\tau_{m} \rightarrow t}$ for a neighbor $m$ as $\bar{g} \cdot \tau_{m}$. To estimate $\underset{(m, w)}{U}{ }^{e}(t)$, however, node $n$ requires some statistics, mentioned in the system model (Section 2). Particularly, the packet at $t-\tau_{m}$ that reached node $n$ from $m$, reports not only $U\left(t-\tau_{m}\right)$, but also its gradient, $\bar{u}$. Thus, $U^{e}(t)$ is estimated as $\max \left\{0, \underset{(m, w)}{U}\left(t-\tau_{m}\right)+\bar{u} \cdot \tau_{m}\right\}$.

Similarly, node $n$ can obtain an estimate of the battery level of node $m, B_{m}^{e}(t)$. This information, coupled with the node's coordinates $\left\{i_{m}, j_{m}\right\},\left\{i_{n}, j_{n}\right\}$ can be employed in a battery and latency-aware policy $\mathcal{P}$. Specifically, the policy specifies the valid neighbors $\mathcal{M}$ of $n$, as the ones that:

- Have a battery level greater than a customized threshold: $B_{m}^{e}(t) \geq B_{T H}$.

- Are nearer to the intended gateway $w$ than node $n$ :

$$
\left|i_{w}-i_{m}\right|+\left|j_{w}-j_{m}\right|<\left|i_{w}-i_{n}\right|+\left|j_{w}-j_{n}\right|
$$

Condition 10 can be ignored when the battery constraint is not met. Finally, by convention, in absence of node statistics batteries are assumed to be fully charged and packet buffers empty. When a node loses power, its estimations are also reset to these default values.

\section{Evaluation}

A HyperSurface is simulated in the Anylogic platform [21, considering both the microscopic (nanonetwork operation) and the macroscopic (interaction with impinging waves) aspects:

Macroscopic setup. We assume the system model of Fig. 3 The HyperSurface has dimensions $Y_{\max }=500 \mathrm{~cm}, Z_{\max }=500 \mathrm{~cm}$. A wave point-source enters at point $\{500,0,500\} \mathrm{cm}$ and moves towards point $\{500,500,0\}$, parallel to the $y-z$ plane with constant velocity $|\vec{v}|=5 \mathrm{~cm} / \mathrm{sec}$. Its emission cone has a direction perpendicular to the $y-z$ plane, and an opening angle of $10^{\circ}$. The emission is isotropic within this cone, and has a constant power of $100 \mathrm{~mW}$. Free-space path loss is the sole attenuation factor.

Microscopic setup. We consider a $31 \times 31$ rectangular grid of asynchronous nano-nodes. The four nodes placed at the middle-most HyperSurface edge points act as gateways and have no power constraints. All other nodes have a power harvesting rate of $10 \mathrm{pJ} / \mathrm{sec}$, an energy drain per (wired) packet emission of $10 \mathrm{pJ}$, and a battery capacity of $1000 \mathrm{pJ}$ [7. Each node senses the impinging power periodically, (input 


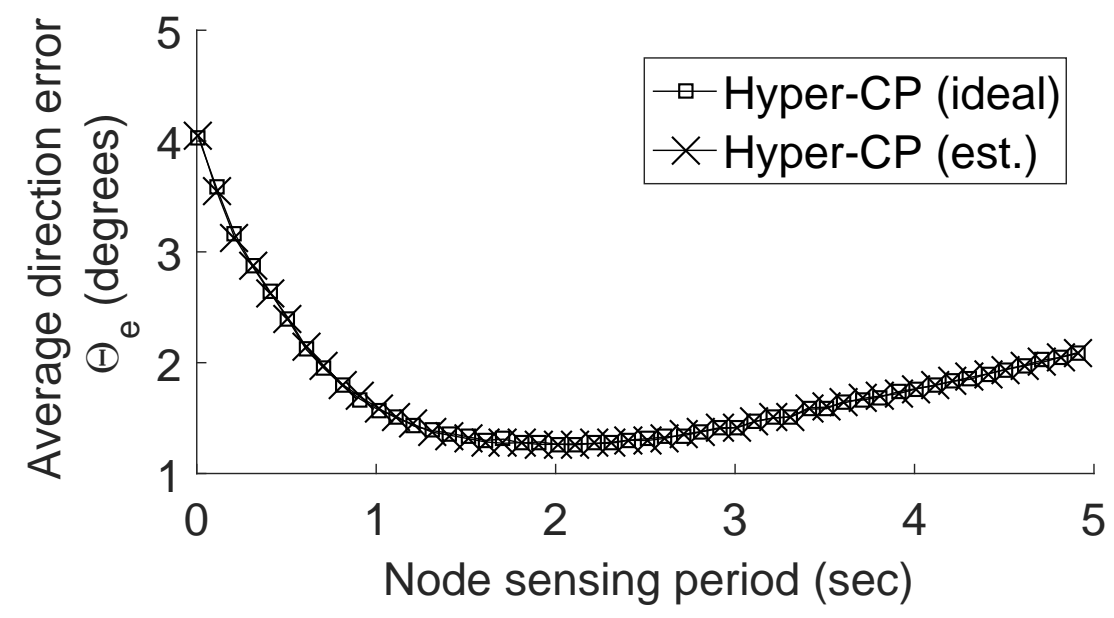

Fig. 4: Hyper-CP direction error versus the node sensing period.

variable sensing period). Zero-power measurements are ignored and do not propagate within the network. Original packets are sent towards their nearest gateway. The total packet propagation time over any link is set to $1 \mathrm{msec}$. The battery threshold $B_{T H}$ is set to $50 \%$ in all cases, but is disregarded if no neighboring node can meet it. Each node has a packet queue that can hold up to 10 packets before overflowing. As a means against power depletion, once a packet queue empties completely, the corresponding node enters a "resting" mode for $1 \mathrm{sec}$, refraining from further packet transmissions to replenish his battery level.

A packet that has reached a gateway is considered to be available to an external server. The server constantly derives the estimated incident point $P^{\prime}$ as the average of the coordinates of the last 50 sensors with a power reading. Moreover, the estimated incident direction $\overrightarrow{d_{I}^{e}}$ is continuously calculated as the conic section that best fits the last 50 power measurements.

Two Hyper-CP variants are compared. The first is given perfect knowledge of the nodes' statuses (ideal), while the second relies on the estimations described in Section 3 The macroscopic performance metrics of interest are the average offset and direction errors throughout the source movement. Microscopic metrics are the corresponding average node battery and packet overflow rate. All runs are repeated 100 times and mean values are presented in the ensuing Figures.

Results. The macroscopic-aspect results are presented in Fig. 4 and 5 , while the corresponding microscopic-aspect performance is shown in Fig. 6 and 7

The $\mathrm{x}$-axis in all Figures represents the node sense period, with separate experiments ran for 0.01 to 5 seconds between successive node sensing events. Intuitively, the sampling period should affect both the macroscopic and microscopic performance. On one hand, a smaller sensing period means more frequent power sampling, thus facilitating the tracking of a moving source. On the other hand, it also leads to an increasing number of generated packets, which need to be routed towards the gateways. This might in turn cause congestion, negatively affecting the macroscopic performance.

The described trade-off is evident in the case of the direction error in Fig. 4 For 


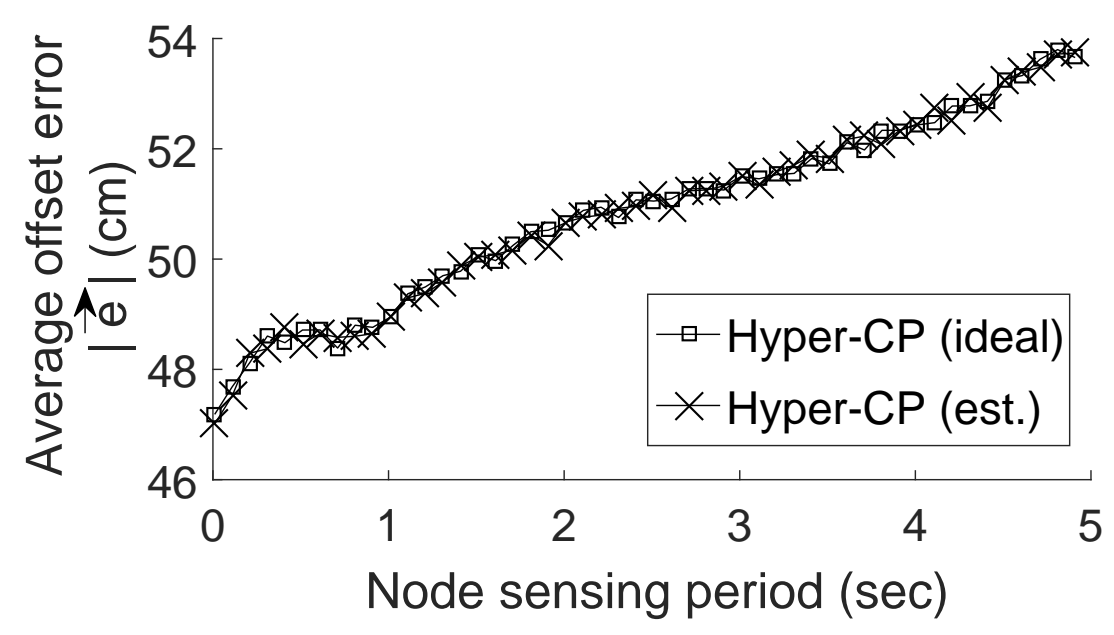

Fig. 5: Hyper-CP offset error versus the node sensing period.

sampling periods of 0.01 to $\sim 1 \mathrm{sec}$, the network is in a congested state, as shown by the packet overflow rate in Fig. 6. The high number of generated packets leads to full packet buffers at the nodes and, thus, to increased buffering times and packet drops, negatively affecting the direction error in Fig. 4. As the sampling period increases, the network stabilizes (Fig. 6, periods $1 \rightarrow 3 \mathrm{sec}$ ) and the direction error reaches a minimal value of approximately $1^{\circ}$. Further increase in the sampling period exhibits a linear increase in the direction error, since the node measurements are not frequent enough to keep track of the moving source. We note that the direction error is an angle metric, and thus generally sensitive to $P^{\prime}$ point estimations. Nonetheless, the estimated direction is generally parallel to the real one, exhibiting a maximum error of $4^{\circ}$.

The offset error, shown in Fig. 5. exhibits a linear increase versus the sampling period, and is not affected by a severe network congestion. The offset error is expressed as the norm of the displacement vector, $\vec{e}$, and thus does not contain error angle information. Moreover, the metric is derived by fitting the measurements incoming to the gateways to the best conic section, as described above. A very limited amount of node measurements is required for this task, and, therefore, a highly congested network state does not have a strong impact on the metric. Thus, the offset error exhibits only a linear increase with the sampling period. Notice that the offset error is moderately high, i.e., $\sim 50 \mathrm{~cm}$, which is the $10 \%$ of the overall space dimensions. However, notice that in conjunction with the minimal direction error discussed above, the estimated source position can be considered as exhibiting a constant displacement with regard to the real position. This means that the external server can employ mobility prediction schemes to mitigate the total macroscopic error.

The average node battery levels, shown in Fig. 7, are naturally expected; smaller sensing periods lead to a higher number of packets to be routed, and eventually to a greater power expense (lower average battery). The node resting mode effectively guards against network energy depletion: The minimum average battery level is $70 \%$ for the minimum sampling period of $0.01 \mathrm{sec}$. Moreover, it increases asymptotically 


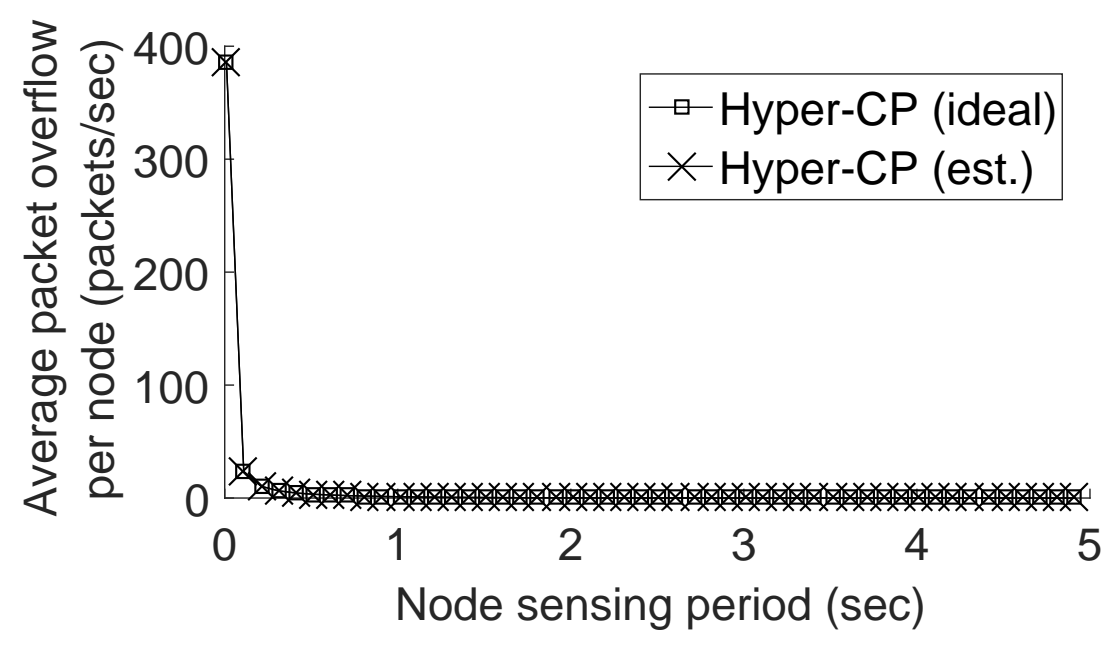

Fig. 6: Node packet overflow rate versus the sensing period.

towards $100 \%$ for higher sensing periods which naturally stress the network less.

Finally, the Hyper-CP protocol is shown to operate equally well with ideal and estimated knowledge of the nodes' status. Queue size estimations may have a negligible effect in the routing decision of relation (9). Assuming that the estimation error is constant (on average) for all nodes, the decision of relation 9 is not significantly affected by it. Additionally, we note that the grid topology also works in favor of the estimations. Each node has a limited number of neighbors (i.e., 4) in the studied grid layout. Moreover, condition (10) may limit the considered nodes further. Thus, routing decision may refer to a limited number of neighbors (e.g., 2) reducing the impact of inaccurate estimations. Wireless network setups, where each node can have multiple neighbors, can constitute a future work direction. The wireless approach can impose a greater energy power consumption to the HyperSurface controller network, but provides the potential for versatility to the HyperSurface deployment, compared to the studied wired approach.

\section{Related Work}

In this Section we review works studying the limitations of nanonetworking in general and related nano-communication protocols.

Wang et al. study manufacturing limitations at nano-scale and highlight the need for unique nano-addressing and nano-routing solutions [20. First, the power supply units of autonomous nano-nodes can scavenge energy for 1 packet transmission per approximately $10 \mathrm{sec}$ [7. Second, a class of wireless nano-communication modules are expected to operate at the $T \mathrm{~Hz}$ band, which translates to highly lossy channel conditions due to acute molecular absorption phenomena [6]. Third, manufacturing restrictions and cost considerations correspond to "weak" nano-node hardware, i.e., limited CPU and data storage capabilities [2, 14. The impact of these restrictions on nano-node addressing is that assigning unique identifiers to each node is not scalable, mainly due to power restrictions. Regarding data routing, on the other hand, 


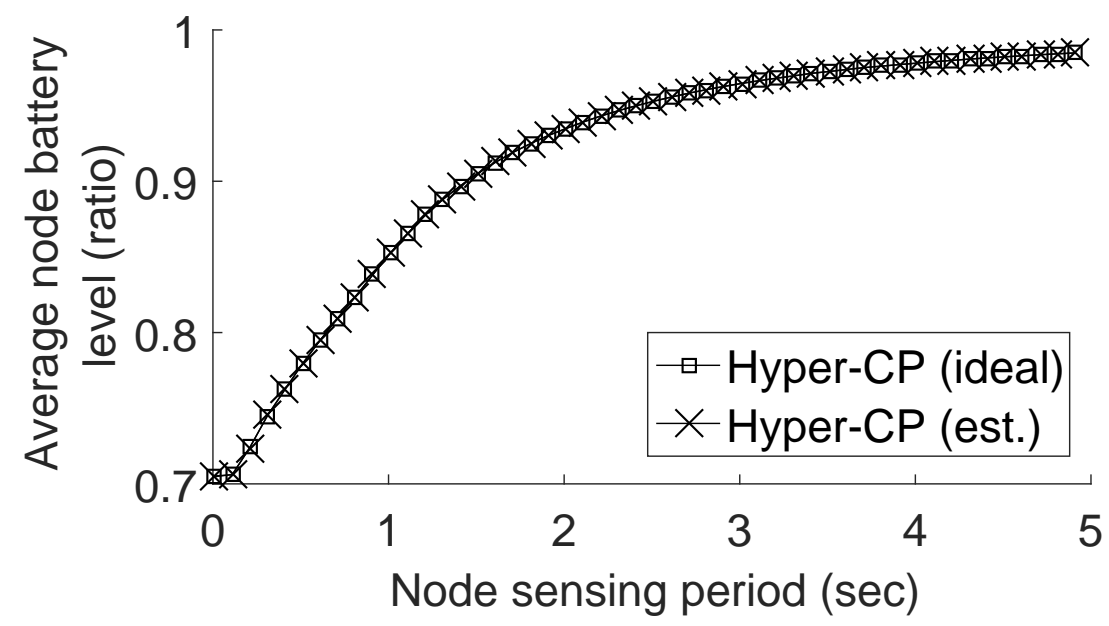

Fig. 7: Average node battery versus the node sensing period.

the expectedly frequent transmission failures require a mechanism to balance path redundancy and energy consumption, while incurring low computational complexity and memory overhead 20. Subsequently, several Medium Access Control (MAC) solutions have been proposed, which assume mobile nodes and $\mathrm{THz}$-operating radio modules [12, 22, These specifications-apart from the strong emphasis on energy efficiency-are not aligned to the described HyperSurface nanonetworks.

Regarding the authors' prior work, a ray-tracing-based simulation technique for nanonetworks was initially studied and validated against precise computational techniques (Finite-Differences Time-Domain) in [8]. Subsequent work focused on application setups were packet flooding constitutes a good match. Liaskos et al. proposed a nature-inspired self-organizing nanonetwork architecture [9]. Tsioliaridou et al. worked towards minimal complexity schemes, expressed by the assumptions of nano-CPUs supporting only basic integer arithmetic, and few bytes of memory [18. A joint peer-to-peer routing and geo-addressing approach for $2 \mathrm{D}$ networks was proposed in [17. The routing component operated via selective flooding contained within network sub-areas (reducing the number of involved retransmitters per communicating node-pair), and with and extremely low computational footprint. The work was then extended to operate in 3D network layouts [19], and by proposing a routing approach over curvilinear paths, essentially minimizing the required retransmitters [16. The present work differentiates by proposing a control protocol specifically for the HyperSurface scenario. The metric of performance is the efficient detection of the direction-of-arrival for waves impinging on the HyperSurface. Moreover, the protocol is point-to-point based, while it considers energy harvesting power supply at the nano-nodes.

\section{Conclusion}

The present work studied nanonetwork-controlled metasurfaces, the HyperSurfaces, which can sense their environment and change their electromagnetic behavior accord- 
ing to external, programmatic commands. A protocol that relays such information must offer natural path diversity, depending on congestion, and nano-node energy shortage. The study proposed the Hyper-CP protocol, which was shown to uphold these conditions via simulations. The Hyper-CP protocol was derived analytically, using a Lyapunov drift minimization approach.

\section{References}

[1] Akyildiz, I., And Jornet, J. The Internet of nano-things. IEEE Wireless Communications 17, 6 (2010), 58-63.

[2] Akyildiz, I. F., And Jornet, J. M. Electromagnetic wireless nanosensor networks. Nano Communication Networks 1, 1 (2010), 3-19.

[3] Georgiadis, L., Neely, M. J., And Tassiulas, L. Resource Allocation and Cross-Layer Control in Wireless Networks. FNT in Networking (Foundations and Trends in Networking) 1, 1 (2005), 1-144.

[4] Holloway, C. L., Kuester, E. F., Gordon, J. A., O’Hara, J., Booth, J., And Smith, D. R. An Overview of the Theory and Applications of Metasurfaces: The Two-Dimensional Equivalents of Metamaterials. IEEE Antennas and Propagation Magazine 54, 2 (2012), 10-35.

[5] Imaszczuk, K., Strikwerda, A. C., Fan, K., Zhang, X., Averitt, R. D., AND JePSEn, P. U. Flexible metamaterial absorbers for stealth applications at terahertz frequencies. Optics Express 20, 1 (2012), 635.

[6] Jornet, J., And Akyildiz, I. Channel Modeling and Capacity Analysis for Electromagnetic Wireless Nanonetworks in the Terahertz Band. IEEE Transactions on Wireless Communications 10, 10 (2011), 3211-3221.

[7] Jornet, J., AND Akyildiz, I. Joint Energy Harvesting and Communication Analysis for Perpetual Wireless Nanosensor Networks in the Terahertz Band. IEEE Transactions on Nanotechnology 11, 3 (2012), 570-580.

[8] Kantelis, K., Amanatiadis, S., Liaskos, C., Kantartzis, N., Konofaos, N., Nicopolitidis, P., And Papadimitriou, G. On the Use of FDTD and Ray-Tracing Schemes in the Nanonetwork Environment. IEEE Communications Letters 18, 10 (2014), 1823-1826.

[9] Liaskos, C., And Tsioliaridou, A. A Promise of Realizable, Ultra-Scalable Communications at nano-Scale: A multi-Modal nano-Machine Architecture. IEEE Transactions on Computers 64, 5 (2015), 1282-1295.

[10] Liaskos, C., Tsioliaridou, A., Pitsillides, A., Akyildiz, I. F., Kantartzis, N., Lalas, A., Dimitropoulos, X., Ioannidis, S., Kafesaki, M., And SoukOulis, C. Design and Development of Software Defined Metamaterials for Nanonetworks. IEEE Circuits and Systems Mag. 15, 4 (2015), 12-25.

[11] Minovich, A. E., Miroshnichenko, A. E., Bykov, A. Y., Murzina, T. V., Neshev, D. N., AND Kivshar, Y. S. Functional and nonlinear optical metasurfaces: Optical metasurfaces. Laser \& Photonics Reviews 9, 2 (2015), 195-213.

[12] Mohrehkesh, S., Weigle, M. C., and Das, S. K. DRIH-MAC: A Distributed Receiver-Initiated Harvesting-Aware MAC for Nanonetworks. Molecular, Biological and Multi-Scale Communications, IEEE Transactions on 1, 1 (2015), 97-110. 
[13] Piro, G., Boggia, G., And Grieco, L. On the design of an energy-harvesting protocol stack for Body Area Nano-NETworks. Nano Communication Networks 6, 2 (2015), 74-84.

[14] Tairin, S., Nurain, N., And Islam,A. B. M. Alim Al. Network-level performance enhancement in wireless nanosensor networks through multi-layer modifications. In 2017 International Conference on Networking, Systems and Security (NSysS), pp. 75-83.

[15] The VISORSURF PROJect. A Hardware Platform for Software-driven Functional Metasurfaces. Horizon 2020 Future Emerging Technologies (2017).

[16] Tsioliaridou, A., Liaskos, C., Dedu, E., And Ioannidis, S. Packet Routing in 3D Nanonetworks: A Lightweight, Linear-path Scheme. Nano Communication Networks PP (2017), PP.

[17] Tsioliaridou, A., Liaskos, C., Ioannidis, S., and Pitsillides, A. COROnA: A Coordinate and Routing system for Nanonetworks. In ACM NANOCOM'15 (2015), p. 18.

[18] Tsioliaridou, A., Liaskos, C., Ioannidis, S., And Pitsillides, A. Lightweight, self-tuning data dissemination for dense nanonetworks. Nano Communication Networks (Special Issue on EM Nanonetworks) 8 (2016), 2-15.

[19] Tsioliaridou, A., Liaskos, C., Pachis, L., Ioannidis, S., And Pitsillides, A. N3: Addressing and Routing in 3D Nanonetworks. In IEEE ICT'16 (2016), pp. $1-6$.

[20] Wang, P., Jornet, J. M., Abbas Malik, M., Fadel, E., And Akyildiz, I. F. Energy and spectrum-aware MAC protocol for perpetual wireless nanosensor networks in the Terahertz Band. Ad Hoc Networks 11, 8 (2013), 2541-2555.

[21] XJ Technologies. The AnyLogic Simulator. 2013.

[22] YaO, X.-W., And Jornet, J. M. TAB-MAC: Assisted beamforming MAC protocol for Terahertz communication networks. Nano Communication Networks 9 (2016), 36-42.

[23] Zhu, A. Y., Kuznetsov, A. I., Luk'yanchuk, B., Engheta, N., And GenEVET, P. Traditional and emerging materials for optical metasurfaces. Nanophotonics 6, 2 (2017). 